\title{
Valoración de los conocimientos que tienen los pacientes en hemodiálisis acerca de su tratamiento
}

\author{
Juan Carlos Sánchez González, Celsa Martínez Martínez, Dámaris Bethencourt Fernández, Marta Pablos López \\ Enfermera/os. Nefrología. Hospital Fundación Jiménez Díaz. Madrid. España
}

\section{Resumen}

Introducción: El incremento de pacientes con enfermedad renal crónica que necesitan de terapias sustitutivas de la función renal es un hecho en nuestro entorno sanitario. La hemodiálisis es uno de los tratamientos sustitutivos de elección para estos pacientes.

Los pacientes presentan con frecuencia dudas y comportamientos que sugieren un déficit de conocimientos sobre la hemodiálisis y los aspectos que la rodean tales como el olvido de la medicación y el desconocimiento de las normas dietéticas entre otros. La falta de dichos conocimientos puede conllevar a la no adherencia del tratamiento lo que constituye un problema sanitario de primer orden.

El objetivo de nuestro trabajo fue analizar el grado de información y conocimientos que tienen los pacientes en programa de hemodiálisis respecto a la propia técnica, a la dieta, a la medicación que deben tomar y al acceso vascular.

Material y métodos: Se realizó un estudio transversal en el que, mediante una encuesta, estudiamos el grado de conocimiento acerca del programa de hemodiálisis y del tratamiento que llevaban a cabo los pacientes con enfermedad renal crónica que recibían hemodiálisis en el Hospital Fundación Jiménez Díaz.

Se evaluaron 32 pacientes, para el análisis estadístico se utilizó la media y desviación típica para las variables cuantitativas y las tablas de contingencia para las variables cualitativas. Para el contraste de hipótesis se empleó el test de la Chi-cuadrado.

Correspondencia:

Juan Carlos Sánchez González

Nefrología. Hospital Fundación Jiménez Díaz

Avda. Reyes Católicos, 2. 28040 Madrid

E-mail: jcsanchez@fjd.es
Resultados: La media de aciertos fue $14,44 \pm 2,75$, de un total de 18 preguntas.

Nuestros pacientes, en general, tienen medios/altos conocimientos, dependientes de edad, ocupación y si cocinan sus propios alimentos.

Conclusiones: en consonancia con otros estudios consideramos que es necesario mantener una educación continua a lo largo del tratamiento con evaluaciones periódicas que nos reporten el nivel de conocimientos a lo largo del tiempo.

\section{PALABRAS CLAVE}

- HEMODIÁLISIS

- TRATAMIENTO FARMACOLÓGICO

- DIETA

- ACCESO VASCULAR

- GRADO DE CONOCIMIENTO

- ENFERMEDAD RENAL CRÓNICA

The assessment of knowledge about treatment in haemodialysis patients

\section{Abstract}

Introduction: The increase in the number of patients with chronic kidney disease (CKD) who require renal replacement therapy is a fact in our healthcare environment. Hemodialysis (HD) is one of the alternative treatments of choice for these patients.

The patients often have doubts and behaviours that suggest a lack of knowledge on hemodialysis and aspects around it such as forgetting medication and ignorance of dietary rules among others. The lack of such knowledge can lead to treatment no adherence what establish a important health problem. 
The aim of our study was to analyze the information and knowledge grade in hemodialyzated patients about the HD, diet, medication and their vascular access (VA).

Material and methods: We did a cross-sectional study in which, with a inquiry, we studied the knowledge grade about hemodialysis treatment in patients with CKD who received hemodialysis at the "Fundación Jiménez Díaz Hospital".

32 patients were evaluated, for statistical analysis the mean and standard deviation for quantitative variables, and frequency table and contingency tables for qualitative variables was used. For hypothesis testing the test of Chi-square test was used.

Results: The successes rate was $14.44 \pm 2.75$, a total of 18 questions.

Our patients generally have mid / high knowledge, dependent on age, occupation and if they cook their own food.

Conclusions: According to others studies we believe to keep a continuous education throughout treatment is essential, in addition to periodic reviews to indicate the knowledge grade.

\section{KEYWORDS}

- HEMODIALYSIS

- DRUG TREATMENT

- DIET

- VASCULAR ACCESS

- KNOWLEDGE GRADE

- CHRONIC KIDNEY DISEASE

\section{Introducción}

La enfermedad renal crónica (ERC) constituye un importante problema de salud en la actualidad, tanto por su elevada prevalencia, como por su importante morbimortalidad cardiovascular. La prevalencia de la ERC ha aumentado de forma progresiva en los últimos años debido principalmente a la optimización de la técnicas depurativas que permiten permanecer más tiempo en la técnica, al envejecimiento de la población y a la alta tasa de enfermedades cardiovasculares, como la diabetes y la hipertensión arterial (HTA $)^{1-2}$. Sin embargo, la incidencia ha disminuido debido a las campañas de prevención llevadas a cabo ${ }^{2}$.

De los tratamientos sustitutivos de la función renal, la hemodiálisis (HD) se emplea en el $80 \%$ de los pacientes con ERC que inician tratamiento renal sustitutivo ${ }^{2-5}$.

Las complicaciones derivadas de la hemodiálisis y de la propia enfermedad se van agravando con el tiempo, percibiendo estos pacientes una forma de vivir más limitada. Esto implica cambios de vida importantes a nivel físico, psicológico y social para el paciente y su familia 6 . Además, dichas complicaciones pueden tener una notable afectación de la calidad de vida si el tratamiento es inadecuado. Por ello, se hace imprescindible evaluar el nivel de conocimientos que sobre la enfermedad tienen los pacientes, pues constituye un método de suma importancia para prevenir las complicaciones y la morbimortalidad en el paciente.

Hace años Virginia Henderson aseguraba que para tener salud era necesario disponer de información, y que no se podía tener salud si no se sabía cómo conservarla, mantenerla o recuperarla ${ }^{7}$.

Una condición básica para que el tratamiento sea favorable es que el paciente se adhiera al mismo y eso sólo se puede conseguir si el paciente interioriza lo que significa ser portador de la enfermedad crónica ${ }^{8}$. Por ello es clave que el paciente tenga conocimientos sobre la enfermedad, siendo la educación sanitaria la mejor herramienta para lograrlo?.

Para que la educación y el aprendizaje sean eficaces se precisa de la participación activa del paciente, con lo que se logrará un adecuado cumplimiento terapéutico, siendo éste el grado en que la conducta del paciente, en relación con la toma de medicación, el seguimiento de una dieta o la modificación del estilo de vida, coincide con la prescripción clínica ${ }^{10}$. Es decir, la aceptación y la implementación por parte del paciente del consejo terapéutico ofrecido. El incumplimiento de éste sería, en parte, responsabilidad del propio paciente y, en parte del personal sanitario, y se relaciona con los conocimientos que éste tenga acerca del tratamiento pautado $0^{10-11}$.

La existencia de cuestiones planteadas a diario por la mayoría de los pacientes sobre la hemodiálisis, la dieta, la medicación y el cuidado del acceso vascular, constituye la razón de este trabajo.

El objetivo principal de este trabajo es valorar el nivel de conocimientos que poseen los pacientes de nuestro centro en relación a su tratamiento.

Como objetivos secundarios nos proponemos ver si variables como edad, sexo, estado civil, nivel de estudios, situación laboral, el haber pasado por la consulta prediálisis, por diálisis peritoneal (DP) o el haber estado 
trasplantado influyen en el nivel de conocimientos que posee el paciente. También si el hecho de cocinar sus propios alimentos mejora los conocimientos en cuanto al tratamiento dietético, o si el poseer un mayor nivel de conocimientos en general, está asociado estar más adaptado a la HD.

Las hipótesis son que los pacientes más jóvenes, con nivel de estudios alto, que están en activo, que han pasado por la consulta prediálisis o diálisis peritoneal o han estado trasplantados previamente tienen mayor nivel de conocimientos. Lo mismo ocurriría con los que son cocineros de sus propios alimentos. Por último aquellos con mayor nivel de conocimientos estarán más adaptados a la HD.

\section{Material y métodos}

Se trata de un estudio transversal, realizado en el servicio de diálisis de la Fundación Jiménez Díaz, siendo éste un hospital terciario de referencia para toda la población de la zona centro de Madrid, que atiende un área de 425.000 habitantes.

Atendiendo a los criterios de selección, que incluían estar en tratamiento de HD, tener una edad superior a 18 años, no tener una patología que impidiera responder a las preguntas o no aceptar participar en el estudio, resultó una muestra de 32 pacientes.

Nuestro tipo de muestreo fue intencional, realizando la encuesta a todos los pacientes de la unidad de diálisis según los criterios de selección de la muestra.

Nuestra herramienta para la recogida de datos de nuestro estudio fue una encuesta (Anexo 1) elaborada por los miembros del equipo basada en una revisión de la literatura. Estaba formada por 21 preguntas, de las cuáles las 20 primeras nos sirven para valorar el grado de conocimiento de los pacientes encuestados y donde se les pregunta acerca de la hemodiálisis (7 preguntas), acceso vascular (2 preguntas), dieta (9 preguntas) y tratamiento farmacológico (2 preguntas) y, por último, la pregunta número 21 que nos servirá para saber si ellos se sienten adaptados a la hemodiálisis y no será tenida en cuenta para medir el grado de conocimiento.

El número máximo de aciertos de la encuesta es de 18, ya que los pacientes con fístula arteriovenosa (FAV) no contestaron a las preguntas 7 y 8 , referidas al catéter, y los pacientes con catéter no contestaron a las preguntas 5 y 6 correspondientes a la FAV.

Para evaluar el grado de conocimiento, valoramos como grado bajo aquellos pacientes que obtuvieron entre $0 \mathrm{y}$
5 aciertos, grado medio entre 6 y 14 aciertos, y grado de conocimiento alto entre 15 y 18 aciertos. De manera más específica, los conocimientos sobre dieta fueron evaluados como altos si tenía 7 ó más preguntas acertadas de un total de 9 , y medios de 3 a 6 aciertos.

La encuesta fue anónima y voluntaria y todos los que la realizaron otorgaron su consentimiento verbal tras haber sido informados. Se realizó entre febrero y abril de 2014, durante las sesiones de HD y no supuso pérdida de tiempo adicional para los pacientes.

La variable principal es el grado de conocimiento de los pacientes. Otras variables estudiadas son: el sexo, la edad, el número de fármacos, si cocina el paciente sus propios alimentos, el nivel de estudios, el estado civil, la ocupación, tipo de acceso vascular que tiene, si ha pasado por la consulta prediálisis, si ha estado en diálisis peritoneal y si ha estado trasplantado.

En cuanto a la variable sobre si cocina el paciente sus propios alimentos se dividió en los que cocinan siempre 0 a veces, y los que no cocinan nunca; por otro lado, se consideraron conocimientos altos si acertaban 7 o más preguntas sobre dieta.

En cuanto al análisis de los datos obtenidos, la descripción de las variables cuantitativas se realizó mediante la media y la desviación estándar, para aquellas que seguían una distribución normal, o mediante la mediana y los cuartiles, para aquellas que no la seguían. La descripción de las variables cualitativas se realizó mediante tablas de contingencia. Para la inferencia estadística, se utilizó el test de la Chi-cuadrado. Los resultados se consideraron significativos para un nivel de significación de 0,05.

Los datos recogidos fueron analizados mediante el paquete estadístico SPSS versión 15.0 para Windows.

Cabe señalar una posible limitación en el estudio como es la muestra, debido a la falta de pacientes que cumplían los criterios de selección en la unidad de diálisis estudiada.

\section{Resultados}

Nuestra muestra de estudio constó de 32 pacientes, 21 hombres y 11 mujeres. El $43,8 \%$ de la muestra tenían una edad comprendida entre 30 y 60 años y el $56,3 \%$ mayor de 60 años. En cuanto al nivel de formación, el $28,1 \%$ poseían formación básica o nula y el $71,9 \%$ formación secundaria o universitaria. También el 34,4\% estaba activo profesionalmente mientras que el $65,6 \%$ estaban ya jubilados. 
El $62,5 \%$ del total de la muestra acudió previamente a la consulta de prediálisis frente a un $37,5 \%$ que no acudió. Un $25 \%$ de nuestros pacientes recibieron previamente diálisis peritoneal frente a un $75 \%$ que no. Por otro lado el $28,1 \%$ habían sido trasplantados mientras que un 71,9 no lo han sido.

La media de fármacos que tomaban los pacientes al día era de $5,81 \pm 3,96$.

Sólo un $28 \%$ de los encuestados cocinaban ellos mismos su comida frente a un $56,3 \%$ que no lo hacían y un $15,6 \%$ que contestaron hacerlo a veces.

El $56,3 \%$ de los pacientes entrevistados tenían como acceso venoso una fistula arteriovenosa y el $43,8 \%$ un catéter.

El $87,5 \%$ de los pacientes de nuestro estudio aseguraron sentirse adaptados a la hemodiálisis frente a un $6,3 \%$ que decían no estarlo. El resto de pacientes $(6,3 \%)$ no sabía si lo estaban o no.

Respecto a la variable principal, el $56,3 \%$ tenían un grado de conocimiento alto mientras que un $43,8 \%$ tenían un grado de conocimiento medio. Ningún paciente tenía un grado de conocimiento bajo.

La media del total de aciertos sobre las preguntas de hemodiálisis es de 6,5 $\pm 0,98$ de 7 preguntas, en aciertos farmacológicos hay una media de $1,31 \pm 0,73$ de 2 preguntas y en aciertos en dieta una media $6,62 \pm 1,51$ de 9 preguntas. La media del total de acierto en la encuesta es de $14,43 \pm 2,74$ de 18 preguntas.

Tras el contraste de hipótesis de las variables de estudio con el grado de conocimiento se obtuvo significación en las variables expuestas en la Tabla 1.

Tabla 1. Variables significativas respecto al grado de conocimiento.

\begin{tabular}{|l|l|l|l|l|}
\hline Variables & Chi cuadrado & Signific. (p) & Riesgo & $\begin{array}{l}\text { Intervalo } \\
\text { de confianza }\end{array}$ \\
\hline Edad & 5,039 & 0,025 & 0,174 & $0,035-0,851$ \\
\hline $\begin{array}{l}\text { Ocupación } \\
\text { Preguntas }\end{array}$ & 4,453 & 0,035 & 6 & $1,033-34,84$ \\
\hline $\begin{array}{l}\text { acertadas } \\
\text { de dieta con } \\
\text { respecto } \\
\text { a si cocinan }\end{array}$ & 5,723 & 0,017 & 0,133 & $0,023-0,777$ \\
\hline
\end{tabular}

Con respecto a la edad, el ser más joven se asocia a un mayor grado de conocimientos $(p=0,025)$. En concreto, los menores de 60 años tienen casi 6 veces más posibilidades de poseer un grado de conocimientos altos que los mayores de 60 años. (Figura 1).

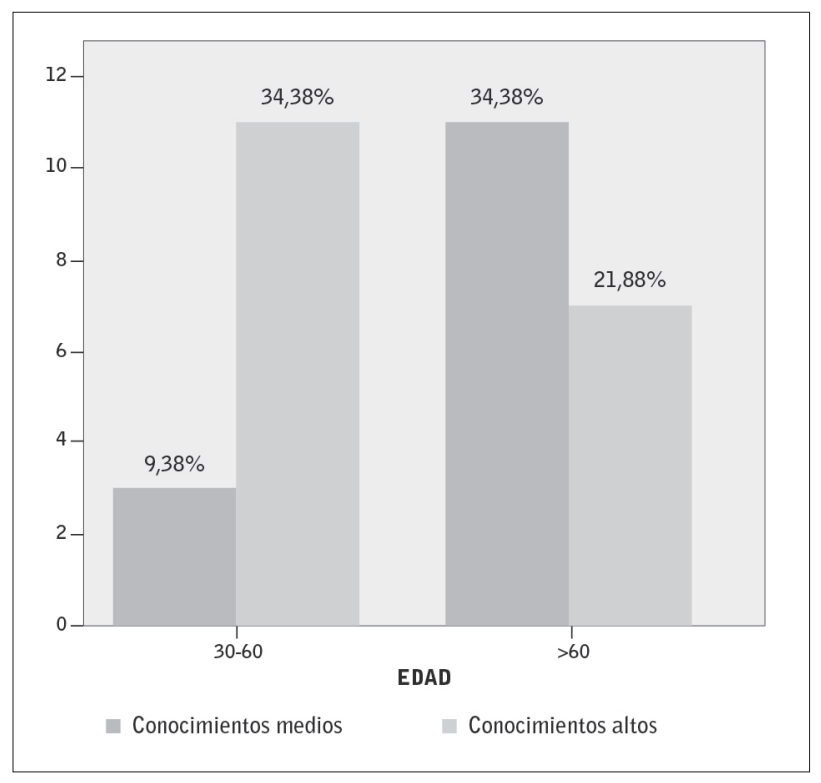

Figura 1. Frecuencias del grado de conocimiento en función de la edad.

El estar laboralmente activo se ha asociado a poseer un mayor grado de conocimiento $(p=0,035)$. En concreto, podemos decir que los pacientes en activo tienen 6 veces más posibilidades de tener un grado de conocimientos alto que los pacientes jubilados. (Figura 2).

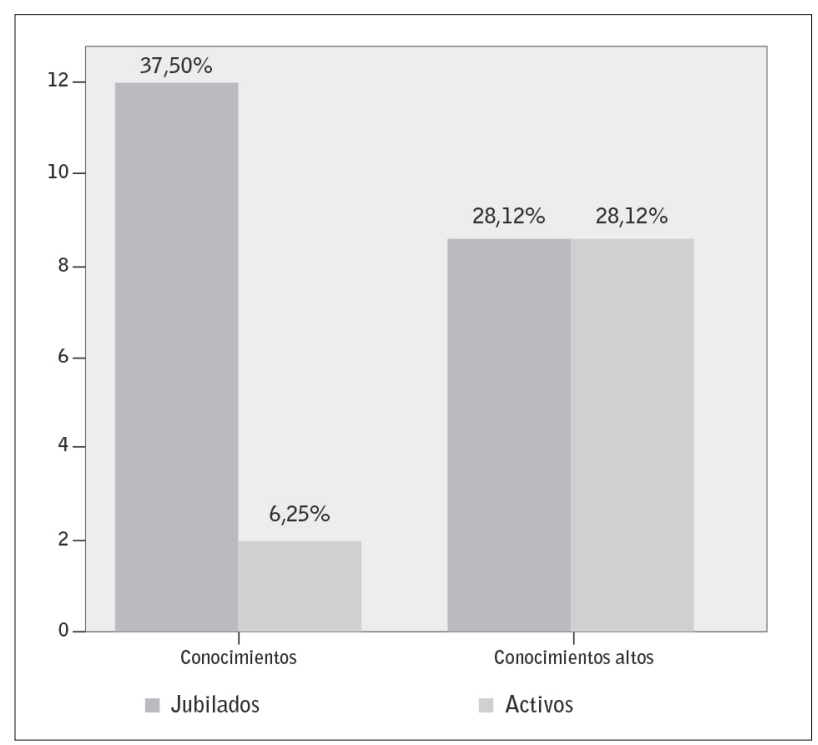

Figura 2. Frecuencias del grado de conocimiento en función de la ocupación.

En cuanto a las preguntas que hacen referencia a la dieta parecen tener más aciertos aquellos que dicen cocinar siempre, 0 a veces, su comida $(p=0,017)$. Es decir que los que cocinan siempre 0 a veces su comida tienen casi 8 veces más posibilidades de tener un grado de conocimientos altos sobre dieta que los que no cocinan nunca. (Figura 3). 


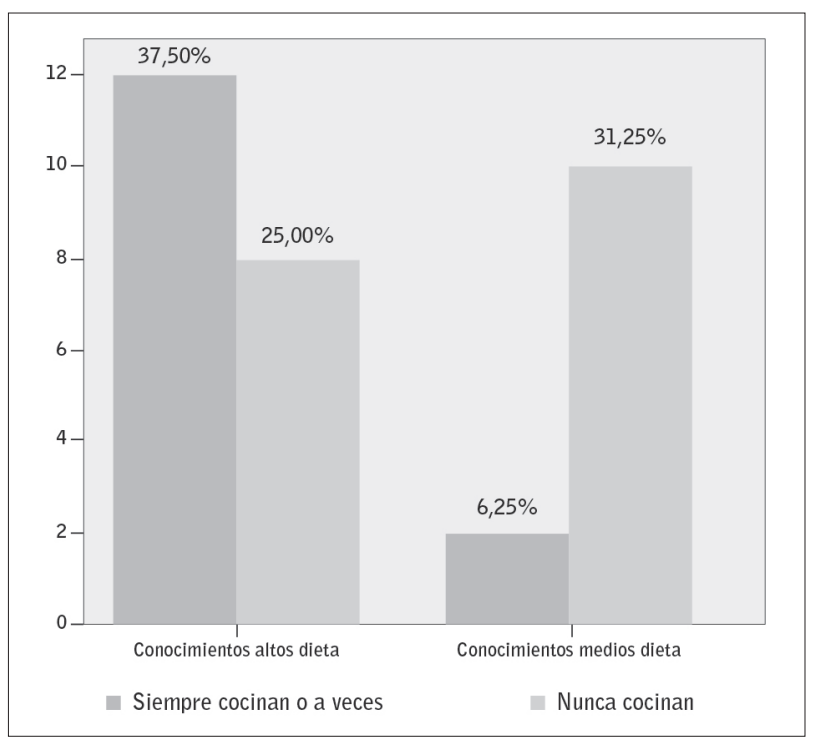

Figura 3. Frecuencias del grado de conocimiento sobre la dieta en función de si cocinan sus propios alimentos.

Las preguntas con menor número de acierto tienen que ver con la dieta. Un $78,1 \%$ responden mal a la pregunta número 13 y un $68,8 \%$ a la pregunta número 14 , ambas referidas al consumo de proteínas.

El 43,8\% responden mal a la pregunta número 19 que hace referencia al tratamiento farmacológico sobre los quelantes del fósforo.

No hay asociación en cuanto a diferencia de conocimientos relativos a fármacos $(p=0,606)$ entre los pacientes que toman mayor número de fármacos frente a los que toman menos.

Sin embargo las preguntas relacionadas con la hemodiálisis han sido las que obtienen un mayor porcentaje de aciertos, y en las que un $71,9 \%$ de los pacientes han respondido bien absolutamente a todas ellas. También las preguntas relacionadas con el acceso vascular, especialmente los portadores de catéter (Figura 4).

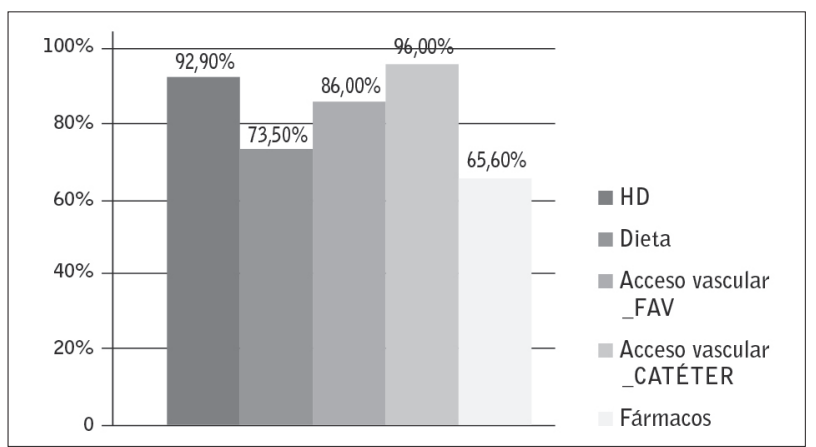

Figura 3. Porcentajes de aciertos de las preguntas de HD, dieta, acceso vascular y fármacos.
No hemos hallado asociación entre haber pasado por la consulta prediálisis y tener un mayor grado de conocimientos $(p=0,854)$ así como tampoco el haber estado en diálisis peritoneal $(p=0,217)$ o el haber estado trasplantado $(p=0,457)$.

En cuanto a si tenía asociación el tener un mayor grado de conocimiento sobre su enfermedad y tratamiento con el sentirse adaptados a la hemodiálisis obtuvimos como resultado que el $42,9 \%$ de los que dicen sí sentirse adaptado en hemodiálisis tienen un grado de conocimiento medio mientras que el $57,1 \%$ tienen un grado de conocimiento alto, no habiendo significación entre sí $(p=0,964)$.

\section{Discusión}

Nos ha llamado la atención que a pesar de ser una población añosa, predomina un nivel de conocimientos medio-alto y que éste no va asociado a haber pasado por consulta prediálisis o por DP o haber sido trasplantado. Probablemente tenga más importancia la educación continua hacia estos pacientes para mantener un adecuado nivel de conocimientos ${ }^{12,13}$.

Como cabía esperar los pacientes más jóvenes y laboralmente activos poseen mayor grado de conocimientos.

Otra cosa a destacar es que no haya significación entre los pacientes que toman mayor número de fármacos frente a los que toman menos en cuanto a conocimientos sobre fármacos se refiere, aunque en este hecho puede influir el escaso número de preguntas que hacen referencia a los fármacos en la encuesta proporcionada.

Por otro lado, aunque existe una asociación entre los que cocinan con el número de aciertos en las preguntas referidas a la dieta, observamos que un gran porcentaje de los pacientes han respondido mal a las preguntas que hacen referencia al consumo de proteínas, un elemento muy importante a tener en cuenta para mantener un buen estado nutricional en pacientes en HD.

La educación para la salud es una herramienta que permite a las personas asumir un rol activo en la modificación de sus conductas y comportamientos para promover la salud, a partir de la incorporación del conocimiento que puede llegar por parte de los profesionales de la salud ${ }^{14}$.

A diferencia del modelo paternalista que se practicaba hace algunos años y en el que era el personal sanitario el que tomaba las decisiones por el paciente, el modelo 
actual basado principalmente en el principio de autonomía otorga al paciente el protagonismo que se merece. Gracias a este cambio de paradigma, la educación no sólo permite que los pacientes aumenten sus conocimientos e ideas acerca de la enfermedad, sino que también mejore su calidad de vida, ya que, en consonancia con otros estudios ${ }^{13}$, el hacer al paciente partícipe de su tratamiento, a través de la educación, mejora la adherencia al tratamiento.

Añadir sobre los estudio de López ${ }^{12}$ y Peláez ${ }^{13}$, que se obtienen resultados diferentes al concluir que los conocimientos no dependen de edad o sexo, por ejemplo, observan que el grado de conocimiento puede variar en función del momento del estudio, del tamaño y tipo de muestra (siendo en el primero una muestra de 110 pacientes y en el segundo 62), pero que tienen en común con el nuestro la idea reforzar periódicamente los conocimientos y llevar a cabo de manera continuada un seguimiento sobre sus necesidades.

Nuestro estudio también difiere de la revisión de Bonilla ${ }^{15}$, según la cual los pacientes que participan en un programa de educación sanitaria específico en una consulta multidisciplinar prediálisis tienen un aumento en su nivel de conocimientos con respecto a los pacientes que no reciben dicha consulta multidisciplinar. Además reconoce que un aumento de conocimientos disminuye el temor y la ansiedad, y aumenta las expectativas de tratamiento y su duración.

Consideramos, por todo esto, que sería conveniente que los profesionales de la salud que realizan educación a personas con ERC estén concienciados de la eficacia que pueden llegar a tener los programas educativos, los cuáles proponemos como uno de los fines de nuestro estudio.

La propuesta sobre diseñar adecuadamente estos programas educativos incide en optimizar el tiempo que los pacientes permanecen en contacto con los profesionales de la salud, durante las sesiones de diálisis, para generar estrategias educativas, y para establecer vínculos con los pacientes para propiciar la educación continua que se necesita para generar un refuerzo en su aprendizaje a corto y largo plazo ${ }^{14}$.

Sería interesante, debido a que una de nuestras limitaciones del estudio ha sido no tener en cuenta el tiempo medio en hemodiálisis de cada paciente, realizar un estudio que evaluase el grado de conocimiento teniendo en cuenta dicha variable para así saber si repercute el tiempo que llevan en tratamiento en diálisis y estudiar así la hipótesis de si tienen más conocimientos aquellos que llevan menos tiempo y han pasado por la consulta de prediálisis y lo tienen más reciente o bien aquellos que llevan un periodo largo en tratamiento teniendo esa consulta más lejana, y por otro lado ver la influencia que tiene el refuerzo educativo por parte propio paciente y del personal profesional de enfermería. Igualmente sería recomendable ampliar el tamaño muestral en sucesivos estudios sobre el tema.

\section{Agradecimientos}

Agradecemos a los pacientes su predisposición y colaboración a la hora de realizar la encuesta, sin ellos este trabajo no habría sido posible.
Recibido: 15 diciembre 2014
Revisado: 15 enero 2015
Modificado: 2 febrero 2015
Aceptado: 12 febrero 2015

\section{Bibliografía}

1. Gándara M., Menezo R., Cobo JL, Pelayo R., García M., Aja A. Valoración de necesidades de las personas con enfermedad renal en hemodiálisis en Cantabria: Rev Nuberos científica Nov 2011 [citado: 22-102013]; 1 (5): Disponible en: http://www.enferme riacantabria.com/enfermeriacantabria/web/articu los/2/12.

2. Sánchez E. Registro Español de Enfermos Renales. Informe de diálisis y trasplante 2012 [Internet]. Oviedo: Registro de Diálisis y Trasplante de la Sociedad Española de Nefrología; 2013 [citado 10 oct 2014]. Disponible en: http://www.senefro.org/mo dules/webstructure/files/reer_datos_2012_sen_bil bao_2013.pdf.

3. Failure K. National Institute of Diabetes and Digestive and Kidney Diseases. Treatment Methods for Kidney Failure: Hemodialysis. Maryland: NKUDIC; 2007.

4. Urzúa A., Pavlov R., Cortés R., Pino V. Factores psicosociales relacionados con la calidad de vida en salud en pacientes hemodializados: Ter Psicol 2011 [citado: 22-10-2013]; 29 (1): 135-140. Disponible en: http://www.scielo.cl/scielo.php?pid=S07184808201 $1000100014 \&$ script=sci_arttext\&tlng=pt. 
5. Soriano S. Definición y clasificación de los estadios de la enfermedad renal crónica. Prevalencia. Claves para el diagnóstico precoz. Factores de riesgo de enfermedad renal crónica: NEFROLOGÍA 2004; 24(6): 27-34.

6. Ruíz de Alegría B.,Basabe Barañano N., Fernández E., Baños C., Nogales MA., Echebarria M., et al. Cambios en las estrategias de afrontamiento en los pacientes de diálisis a largo tiempo. Rev Soc Esp Enferm Nefrol 2009; 12(1): 11-17.

7. Pascual $R$, Andreu L. Programa de educación sanitaria del paciente en hemodiálisis. Rev Soc Esp Enferm Nefrol 1989.

8. Noda JR., Pérez JE., Málaga G., Aphang Lam R. Conocimientos sobre su enfermedad en pacientes con diabetes mellitus tipo II que acuden a hospitales generales: Rev. Med Hered 2008 [citado: 28-10-2013]; 19 (2): 68-72. Disponible en: http://www.scielo.org.pe/scielo.php?pid=S1018130X2008000200005\&script=sci_arttext.

9. Guerra VT., Díaz AE., Vidal K. La educación como estrategia para mejorar la adherencia de los pacientes en terapia dialítica: Rev Cubana Enfermer 2010 [citado:21-10-2013]; 26(2). Disponible en: http://scielo.sld.cu/scielo.php?script=sci_arttext\&pid $=$ S0864-03192010000200007.

10. Sáez S, Domingo E, Martí A, Moreno C, Muñoz $A$, Ortells $R$. Impacto de la información recibida en el conocimiento del tratamiento farmacológico prescrito a pacientes en diálisis. Libro Comunicaciones presentadas al XXXI congreso nacional de la sociedad española de enfermería nefrológica [Internet]. 2006 [citado 22-10-2013]: 195-204. Disponible en: http://www.revistaseden.org/files/195.pdf.

11. Vila M, García N, Gómez A, García R, Tejuca M, Tejuca $A$. Cumplimentación terapeútica de pacientes en diálisis. Libro Comunicaciones Presentadas al XXXII Congreso Nacional SEDEN [Internet]. 2007 :173-7. Disponible en: http://www.revistaseden.org/ files/1822_h8.pdf.

12. López JL., Tomás M., Pujol S. Conocimientos del enfermo renal en hemodiálisis. Libro Comunicaciones XXXV Congreso Nacional SEDEN [Internet]. 2010 [citado....]:113-6. Disponible en: http://www.revis taseden.org/files/3353_2010redu.pdf.

13. Peláez B., Fernández M., Núñez M., González I., Méndez A., Quinatana A. Evaluación de los conocimientos prácticos de los pacientes prevalentes en diálisis peritoneal. Rev Soc Esp Enferm Nefrol 2013; 16(3): 179-184.

14. Guerra VT., Díaz AE., Vidal K. La educación como estrategia para mejorar la adherencia de los pacientes en terapia dialítica. Rev Cub Enferm 2010; 26(2).

15. Bonilla FJ. Educación sanitaria al paciente con enfermedad renal crónica avanzada. ¿Existe evidencia de su utilidad?. Rev Soc Esp Enferm Nefrol 2014; 17(2): 120-31.

\section{Anexo 1.}

\section{ENCUESTA}

Sexo:

Hombre $\square \quad$ Mujer $\square$

Edad:

18-30 años $\square \quad$ 30-60 años $\square \quad+$ de 60 años $\square$

Estudios:

Sin formación $\square \quad$ Formación básica $\square$

Formación secundaria $\square \quad$ Formación universitaria $\square$

Estado civil:

Soltero/a $\square \quad$ Casado/a $\square \quad$ Viudo/a $\square$

Ocupación:

¿Ha pasado por la consulta de pre-diálisis? Si $\square \quad$ No $\square$

¿Ha estado anteriormente en diálisis peritoneal? Si $\square \quad$ No $\square$

¿Ha sido trasplantado? Si $\sqcup \quad$ No $L$

Número de fármacos que toma:

Cocinero de sus propios alimentos: Si $\square \quad$ Si $\square \quad$ A veces $\square$

Tipo de acceso vascular: Fistula $\square \quad$ Injerto $\square \quad$ Catéter $\square$

1. ¿Sabe cuáles son las funciones del riñón?

a) Es el encargado de repartir la sangre por el cuerpo

b) Limpiar sustancias tóxicas del organismo y eliminar líquidos a través de la orina

c) Absorber los nutrientes de mi alimentación

2. ¿Sabe que es la Insuficiencia Renal Crónica?

a) Fallo de las funciones del riñón

b) Orinar mucha cantidad

c) Orinar sólo por la noche

3. ¿Qué es la Hemodiálisis?

a) Tratamiento que permite curar la ERC en unas pocas sesiones

b) Tratamiento que se realiza a través del abdomen

c) Es un procedimiento destinado a suplir la función depuradora del riñón

4. ¿Sabe usted por qué le pesan siempre antes de comenzar cada sesión de Hemodiálisis?

a) Para saber su peso acumulado desde la anterior sesión de diálisis y así poder programar los parámetros de su tratamiento 
b) Para saber si estoy o no desnutrido

c) Para saber si hay que ponerse a dieta

\section{5. ¿Qué es la fístula arterio-venosa?}

a) Es un bulto en la piel

b) El acceso permanente, dónde se une una arteria y una vena, normalmente en el brazo, a través del cual su sangre es llevada a la máquina de diálisis

c) Es un tubo de gran calibre, insertado en el cuello, para extraer la sangre en la Hemodiálisis

\section{6. ¿Qué cuidados debe darle a la fístula arterio-venosa?}

a) Le pueden tomar la tensión y sacarle sangre en el brazo portador de la fístula

b) No le pueden tomar la tensión ni sacar sangre del brazo portador de la FAVI, no coger peso, no llevar ropa u objetos apretados y debe mantener una buena higiene diaria

c) No me la puedo mojar al ducharme

\section{7. ¿Qué es el catéter?}

a) Es un tubo de plástico que se introduce en un vaso sanguíneo de gran calibre para extraer la sangre y realizar la hemodiálisis

b) Es una vía que me cogen para meter medicación

c) Unión de arteria y vena en el brazo que permita realizar la hemodiálisis

\section{Con respecto al cuidado del catéter indique cuál es verda-} dera:

a) Realizar la ducha con precaución para mantener el catéter protegido y seco, y comunicar a la enfermera la aparición de dolor y calor en esta zona

b) Puede utilizarlo para otros tipos de tratamientos ajenos a la hemodiálisis

c) No tiene importancia que se le moje

9. ¿Cuál de estos alimentos es recomendable por su bajo contenido en potasio para su dieta?

a) Plátano, kiwi, naranja

b) Pepino, lechuga y compota de manzana

c) Lentejas

\section{0. ¿Cómo debe cocinar sus verduras?}

a) Cortando la verdura en trozos pequeños, dejándola en remojo un mínimo de tres horas y cambiando el agua varias veces sin consumir el agua del remojo, la verdura congelada pierde potasio al dejarla descongelar a temperatura ambiente

b) Cocinándola poco tiempo

c) Echándole mucha sal

11. Como sabe, abusar de alimentos ricos en potasio puede poner en riesgo su vida, ¿sabe qué síntomas podría dar un consumo elevado de potasio y ante los cuales debería ir a Urgencias inmediatamente?

a) Cuándo presente cansancio, hormigueos, pesadez en brazos y piernas y dificultad para hablar

b) Cuando presente fiebre y congestión nasal

c) Cuándo presente dolor de oído

12. Como sabe, una de las formas de reducir el fósforo en sangre es reducir su consumo. ¿sabe qué alimentos son ricos en fósforo?
a) Zanahoria

b) El queso, el yogur, alimentos con harina como pan, tallarines o productos de pastelería y la coca-cola

c) Espárragos

13. ¿Es necesario que disminuya el consumo de proteínas en su tratamiento de diálisis?

a) No ni antes ni durante el tratamiento

b) No, sólo al comenzar el tratamiento en diálisis

c) Puedo comer todas las proteínas que quiera

14. ¿Cómo debe ser la ingesta de proteínas en un paciente en HD? a) Menor para no forzar al riñón

b) Mayor porque se pierden proteínas durante el tratamiento con hemodiálisis

c) Es indiferente el consumo de proteínas que se realice en la dieta

15. ¿Cuál es la ingesta de líquidos recomendada en Hemodiálisis?

a) No puedo beber líquidos

b) Medio litro + cantidad que orine en $\mathbf{2 4}$ horas

c) Puedo beber lo que quiera

16. Una posible complicación en pacientes en HD es el llamado edema agudo de pulmón, que se puede producir al ingerir más líquido del recomendado, ¿qué síntomas puede sentir y ante los cuales debería acudir rápidamente a Urgencias?

a) Dificultad respiratoria, hinchazón en cara, brazos y piernas

b) Fatiga y vómitos

c) Dolor de cabeza

17. ¿Cómo debe actuar para no sobrepasar la ingesta de líquido?

a) Saltear las verduras antes de hervirlas

b) No beber nada de agua

c) Limitar la ingesta de comidas saladas ya que aumenta la sed y medir el líquido total que debo tomar al día

18. ¿La EPO está incluida en su medicación?

a) No

b) La EPO me la aporta la dieta

c) Si ya que ésta hormona es producida por el riñón y debido a mi enfermedad esta producción se ve disminuida

19. ¿cuál de estos medicamentos se utiliza para disminuir el fósforo en la sangre?

a) Caosina, Royen, Renagel, Fosrenol, Renvela

b) Enalapril, EPO, hierro, Augmentine

c) Resin calcio, resin sodio, adiro, Sintrom

20. ¿Cree usted que la Hemodiálisis es un tratamiento curativo?

a) Sí, con unas cuantas sesiones los riñones se curarán y no tendré que volver a hemodiálisis

b) No, pero ayuda a mejorar la calidad de vida

c) No y no sirve para nada

21. ¿Cree usted que está adaptado a la hemodiálisis?
a) $\mathrm{Si}$
b) No
c) No sé 